\title{
Impact of Ultrasonic Scalpels for Liver Parenchymal Transection on Postoperative Bleeding and Bile Leakage
}

\author{
IOANNIS D. KOSTAKIS, NIKOLAOS MACHAIRAS, ZOE GAROUFALIA, \\ ANASTASIA PRODROMIDOU and GEORGIOS C. SOTIROPOULOS

\begin{abstract}
Second Department of Propaedeutic Surgery, "Laiko" General Hospital, Medical School,
\end{abstract} \\ National and Kapodistrian University of Athens, Athens, Greece
}

\begin{abstract}
Background/Aim: Novel techniques for liver parenchymal transection have emerged and they are available to the hepatobiliary surgeon. The aim of our study was to compare two types of ultrasonic scalpels (Lotus and Harmonic) and examine how they perform either alone or in combination with the SonaStar ultrasonic surgical aspiration system regarding postoperative bleeding and bile leakage. Patients and Methods: Our prospectively maintained database of patients who underwent liver resections in our Department was reviewed. One hundred and two patients with solid liver lesions underwent liver resection by a senior hepatobiliary surgeon in our department during a period of 51 months. They were divided into four groups according to the devices that were used for liver parenchymal transection. Results: Patients were divided into the following groups: group 1: Lotus, 32 patients (31.4\%); group 2: Lotus+SonaStar, 27 patients (26.5\%); group 3: Harmonic, 27 patients (26.5\%); group 4: Harmonic+SonaStar, 16 patients (15.7\%). There were 5 cases of postoperative bleeding and 9 cases of postoperative bile leakage. No significant difference was found concerning postoperative bleeding (group 1: $2 / 32 ; 6.3 \%$, group $2: 2 / 27$; $7.4 \%$, group 3: 0/27; 0\%, group 4: $1 / 16 ; 6.3 \%)(p=0.577)$. Furthermore, no actual difference was detected in terms of postoperative bile leakage (group 1: 2/32; $6.3 \%$, group 2: $3 / 27 ; 11.1 \%$, group $3: 3 / 27 ; 11.1 \%$, group $4: 1 / 16 ; 6.3 \%)$ $(p=0.866)$. Conclusion: Both Lotus and Harmonic ultrasonic
\end{abstract}

This article is freely accessible online.

Correspondence to: Ioannis D. Kostakis, MD, MSc, Ph.D., Second Department of Propaedeutic Surgery, "Laiko" General Hospital, Medical School, National and Kapodistrian University of Athens, 17 Agiou Thoma Street, 11527, Athens, Greece. Tel: +30 6974906246, +30 2107709948, Fax: +30 2107709949, e-mail: i.d.kostakis@gmail.com

Key Words: Liver parenchymal transection, liver resection, hepatectomy, ultrasonic scalpel, Lotus, Harmonic, bile leakage, bleeding. scalpels provide adequate and similar results concerning postoperative hemorrhage and cholorrhea.

Hepatic resections are acknowledged as highly challenging surgical procedures due to the particular biliary and vascular hepatic anatomy, eventual underlying chronic liver diseases and potentially hard-to-control bleeding from parenchymal transection. As the technology of surgical instruments and equipment advances, new modalities have been added in the arsenal of hepatobiliary surgeons for the treatment of liver tumors. Novel techniques for liver parenchymal transection have emerged and they are available to the hepatobiliary surgeon, along with the old technique of clamp-crush. Some of the most used modalities that have been tested for liver resection are ultrasonic aspirator systems (e.g. cavitron ultrasonic surgical aspirator (CUSA)), ultrasonic scalpels (e.g. Harmonic, Lotus), vessel-sealing systems (e.g. LigaSure, Enseal), dissection through radiofrequencies (e.g. (radiofrequency dissecting sealer (RFDS)), saline-linked radiofrequency sealer (SLRS) (e.g. Salient Dissecting Sealer), hydrojet and vascular staplers (1-9). Most studies and metaanalyses focusing on the impact of the exact type of liver parenchymal transection method on intraoperative and postoperative outcomes compare the novel modalities with the old clamp-crush technique mainly and the various categories of new techniques with each other secondarily $(1,5,6,10-14)$.

The objective of our study was to compare two types of ultrasonic scalpels, the Lotus (laparoscopic operation by torsional ultrasound) ultrasonic scalpel and the Harmonic ultrasonic scalpel, and to examine how they perform either alone or in combination with the SonaStar ultrasonic surgical aspiration system, focusing on the occurrence of postoperative bleeding and bile leakage.

\section{Patients and Methods}

Patients. We reviewed our prospectively maintained database of patients who underwent liver resections in our department. One hundred and two patients with solid liver lesions underwent liver 
resection by a senior hepatobiliary surgeon in our department during a period of 51 months. Our database was reviewed regarding patients' gender, age and body mass index (BMI), presence of liver cirrhosis, type of tumor, number of lesions, size of lesions, the hepatic segments that were infiltrated by the tumor, the exact type of hepatic resection and whether it corresponded to major hepatectomy ( $\geq 3$ liver segments), whether the operation was performed open or laparoscopically, duration of vascular occlusion (Pringle maneuver or total vascular occlusion), total operating time, devices used for liver parenchymal transection, Dindo-Clavien classification of surgical complications (15) and presence of postoperative bleeding or bile leakage.

The patients were divided into four groups according to the devices that were used for liver parenchymal transection. The first group included hepatectomies performed with Lotus ultrasonic scalpel (Lotus Liver Resector, BOWA-electronic GmbH \& Co. KG, Gomaringen, Tübingen, Germany) alone, the second group included hepatectomies performed with Lotus Liver Resector and SonaStar ultrasonic surgical aspiration system (Misonix Inc., Farmingdale, NY, USA), the third group included hepatectomies performed with Harmonic ultrasonic scalpel (Harmonic ACE +7 Shears, Ethicon Inc., Somerville, NJ, USA) alone and the fourth group included hepatectomies performed with Harmonic ACE +7 Shears and SonaStar ultrasonic surgical aspiration system. This study conforms to the Declaration of Helsinki and the guidelines of the Ethical Committee of our institution. All patients gave their informed consent to participate in this study.

Statistical analysis. Chi-square test and Fisher's exact test were used for comparisons among groups with categorical variables. Logistic regression analysis was used for multivariate analysis when the dependent outcome was a categorical dichotomous variable. All the tests were two-tailed. Results were considered statistically significant if the $p$-value was less than 0.05 .

\section{Results}

Patients' characteristics. One hundred and two patients (61 men and 41 women) underwent liver resection for solid hepatic lesions. The mean age was 60.6 years (SD: 12.8 years) and the median age was 62 years (min-max: 19-86 years). The first group (Lotus) included 32 patients $(31.4 \%)$, the second (Lotus+SonaStar) 27 patients $(26.5 \%)$, the third (Harmonic) 27 patients $(26.5 \%)$ and the fourth (Harmonic+SonaStar) 16 patients (15.7\%). Table I shows patients' and tumor characteristics, as well as intraoperative and postoperative data.

Impact of liver parenchymal transection technique on postoperative bleeding. Five cases $(4.9 \%)$ of postoperative bleeding were recorded, which were treated conservatively with transfusion of blood and fresh frozen plasma, thus representing a grade II surgical complication according to the Dindo-Clavien classification (13). No significant impact of liver parenchymal transection technique on postoperative bleeding was found. In particular, no significant difference was detected when the four groups were compared to each
Table I. Patients', tumor, intraoperative and postoperative data.

\begin{tabular}{|c|c|}
\hline Gender & Male: 61 , Female: 41 \\
\hline \multirow[t]{2}{*}{ Age } & Mean \pm SD: 60.6 years \pm 12.8 \\
\hline & Median (min-max): 62 years $(19-86)$ \\
\hline \multirow[t]{2}{*}{ Body mass index (BMI) } & Mean \pm SD: $26.4 \pm 3.7$ \\
\hline & Median (min-max): $26(17.2-38.3)$ \\
\hline Liver cirrhosis & Yes: 10, No: 92 \\
\hline \multirow[t]{6}{*}{ Type of tumor } & Hepatocellular carcinoma: 33 \\
\hline & Cholangiocarcinoma: 21 \\
\hline & Liver metastases: 40 \\
\hline & Liver cell adenoma: 3 \\
\hline & Focal nodular hyperplasia: 1 \\
\hline & Hemangioma: 4 \\
\hline Number of lesions & Solitary: 65 , Multiple: 37 \\
\hline \multirow[t]{2}{*}{ Maximum tumor diameter } & Mean \pm SD: $5.1 \mathrm{~cm} \pm 3.9$ \\
\hline & Median (min-max): $4.2 \mathrm{~cm}(0.4-18.3)$ \\
\hline Major hepatectomy & Yes: 25, No: 77 \\
\hline Type of operation & Open: 81, Laparoscopic: 21 \\
\hline Pringle maneuver & Yes: 54 , No: 48 \\
\hline \multirow{2}{*}{$\begin{array}{l}\text { Duration of Pringle } \\
\text { maneuver }\end{array}$} & Mean \pm SD: 13.4 minutes \pm 15.8 \\
\hline & Median (min-max): 8 minutes $(0-60)$ \\
\hline \multirow[t]{2}{*}{ Total operating time } & Mean \pm SD: 195.8 minutes \pm 70.5 \\
\hline & Median (min-max): 190 minutes $(60-420)$ \\
\hline \multirow[t]{4}{*}{ Devices } & Lotus: 32 \\
\hline & Lotus + SonaStar: 27 \\
\hline & Harmonic: 27 \\
\hline & Harmonic + SonaStar: 16 \\
\hline $\begin{array}{l}\text { Surgical complication } \\
\geq \text { grade III }\end{array}$ & Yes: 11, No: 91 \\
\hline Postoperative bleeding & Yes: 5, No: 97 \\
\hline Postoperative bile leakage & Yes: 9 , No: 93 \\
\hline
\end{tabular}

other $(p=0.577)$, since 2 cases of postoperative bleeding were noted in group $1(2 / 32 ; 6.3 \%), 2$ cases in group $2(2 / 27$; $7.4 \%)$, no case in group $3(0 / 27 ; 0 \%)$ and 1 case in group 4 $(1 / 16 ; 6.3 \%)$. Table II shows the incidence of postoperative bleeding among the different groups.

Impact of liver parenchymal transection technique on postoperative bile leakage. Nine cases $(8.8 \%)$ of postoperative bile leakage were recorded, which were treated with some form of intervention, but without requiring general anesthesia [grade IIIA surgical complication according to the Dindo-Clavien classification (13)], namely percutaneous drainage of biloma (4 cases), percutaneous transhepatic cholangiography (PTC) (3 cases), combined percutaneous drainage of biloma and PTC (1 case) or endoscopic retrograde cholangiopancreatography (ERCP) (1 case).

No significant impact of liver parenchymal transection technique on postoperative bile leakage was found. In particular, no significant difference was detected when the four groups were compared to each other $(p=0.866)$, since 2 cases were observed in group $1(2 / 32 ; 6.3 \%), 3$ cases in group $2(3 / 27 ; 11.1 \%), 3$ cases in group $3(3 / 27 ; 11.1 \%)$ and 
Table II. Postoperative bleeding and bile leakage.

\begin{tabular}{lccccc}
\hline & Lotus & Lotus+SonaStar & Harmonic & Harmonic+SonaStar & $p$-Value \\
\hline Bleeding & $2 / 32$ & $2 / 27$ & $0 / 27$ & $1 / 16$ & 0.577 \\
Bile leakage & $2 / 32$ & $3 / 27$ & $3 / 27$ & $1 / 16$ & 0.866 \\
\hline
\end{tabular}

1 case in group $4(1 / 16 ; 6.3 \%)$. Table II shows the incidence of postoperative bile leakage among the different groups.

\section{Discussion}

Although liver surgery has become more widespread and new modalities have been added to the surgical instruments that the hepatobiliary surgeon has at his/her disposal, the rates of complications after hepatic resections remain relatively high, with mortality ranging between $0.3 \%$ and $9.7 \%$ and morbidity ranging between $4.1 \%$ and $47.7 \%$ in the various studies addressing this issue. The high levels of morbidity and mortality after hepatectomies that have been reported in several studies can be attributed, at least partly, to the advance of surgical techniques and the expansion of criteria for liver resection. Due to them, hepatobiliary surgeons adopt more technically challenging procedures, which are directed towards parenchymal-sparing operations and in many cases are applied to frail patients. Common postoperative complications include fever, subphrenic abscess, pleural effusion, ascites, coagulation disorders, intraperitoneal bleeding, hemobilia, bile leakage, etc. $(16,17)$. Specifically, for postoperative bleeding and bile leakage, the reported incidence is $4.2 \%-10 \%$ and $4 \%-17 \%$, respectively (16-19).

Initially, liver parenchymal dissection was performed without sophisticated modalities, mainly using the clampcrush technique, in which surgical clamps were used to crush liver parenchyma, in order for small blood vessels and bile ducts to become exposed and ligated. However, new techniques for liver parenchymal transection have emerged and are available to the hepatobiliary surgeon, such as ultrasonic aspirator systems (e.g. CUSA), ultrasonic scalpels (e.g. Harmonic, Lotus), vessel-sealing systems (e.g. LigaSure, Enseal), dissection through radiofrequencies (e.g. RFDS), saline-linked radiofrequency sealer (SLRS) (e.g. Salient Dissecting Sealer), hydrojet and vascular staplers (1-9). CUSA (Tyco Healthcare, Mansfield, MA, USA) and SonaStar combine the energy created by ultrasounds with aspiration for the transection of hepatic parenchyma and skeletonization of small blood vessels and bile ducts, which then can be ligated. Ultrasonic scalpels, such as Harmonic and Lotus, use vibrating ultrasonic shears to coagulate and cut tissues. Thus, they seal and divide small blood vessels and transect hepatic parenchyma. Vessel-sealing systems, such as LigaSure
(Covidien, Mansfield, MA, USA) and Enseal (Ethicon Inc., Somerville, NJ, USA), occlude blood vessels and divide liver parenchyma by combining pressure with bipolar diathermy. On the other hand, RDFS uses radiofrequencies to thermocoagulate liver parenchyma, which is transected afterwards $(2,4,7)$. Another type of surgical instrument is the Salient Dissecting Sealer (Salient Surgical Technologies, Portsmouth, NH, USA), formerly known as TissueLink, with which blunt parenchymal dissection and seal of small vessels are achieved by using radiofrequencies and cool saline as a conductor. Furthermore, dissection with hydrojet is another technique using water jet with high pressure, which breaks apart hepatic parenchyma and skeletonizes small blood vessels and bile ducts $(2,7)$. Finally, vascular staplers are also used for liver parenchymal transection, along with division of major blood vessels (2, 4, 7-9).

Several studies have been conducted and evaluated the impact of the exact type of liver parenchymal transection method on intraoperative and postoperative outcomes $(1,5$, $6,10-14)$. However, most studies and meta-analyses have focused on comparing the novel modalities with the old clamp-crush technique mainly and the various categories of new techniques with each other secondarily. As far as postoperative bleeding and bile leakage are concerned, data can be collected by several original studies and metaanalyses $(1,5,6,10-14)$. A meta-analysis conducted by Alexiou et al. found no significant differences between CUSA and RFDS on one hand and the clamp-crush technique on the other hand in regards to postoperative bile leakage. On the contrary, vessel-sealing systems seemed to have lower risk for postoperative cholorrhea when compared with the clamp-crush technique (1). However, in a metaanalysis conducted by Rahbari et al., no actual differences were detected in terms of postoperative bile leakage when the clamp-crush technique was compared with ultrasonic scalpels, vessel-sealing systems, RFDS, saline-linked radiofrequency sealer and hydrojet. The same meta-analysis also showed that the clamp-crush technique has lower transfusion rates than dissection with hydrojet, but no significant differences with ultrasonic scalpels, vessel-sealing systems, RFDS, or saline-linked radiofrequency sealer concerning postoperative bleeding (6). Furthermore, Aoki et al., Bodzin et al. and Appéré et al. reported that there is no actual difference between CUSA and Harmonic ultrasonic 
scalpel $(10,13,14)$. Moreover, Mbah et al. detected no significant difference between Enseal and Harmonic ultrasonic scalpel regarding postoperative hemorrhage or cholorrhea (12). Finally, Richter et al. found no difference in terms of postoperative hemorrhage or cholorrhea when they compared CUSA, hydrojet and SLRS (11).

Our study is the first attempt to compare two different surgical instruments of the same category, namely ultrasonic scalpels, in terms of postoperative bleeding and bile leakage, and to examine their results when they are used alone or in combination with an ultrasonic surgical aspiration system. Our results suggest that Harmonic and Lotus ultrasonic scalpels do not differ in the rates of postoperative bleeding and bile leakage when used either alone or combined with the SonaStar ultrasonic surgical aspiration system. Further studies are needed in order to identify the most suitable surgical instruments and equipment for liver parenchymal transection that result in lower rates of postoperative bleeding and bile leakage.

\section{Conflicts of Interest}

There are no conflicts of interest and there is nothing to declare.

\section{References}

1 Alexiou VG, Tsitsias T, Mavros MN, Robertson GS and Pawlik TM: Technology-assisted versus clamp-crush liver resection: a systematic review and meta-analysis. Surg Innov 20: 414-428, 2013.

2 Otsuka Y, Kaneko H, Cleary SP, Buell JF, Cai X and Wakabayashi G: What is the best technique in parenchymal transection in laparoscopic liver resection? Comprehensive review for the clinical question on the 2nd International Consensus Conference on Laparoscopic Liver Resection. J Hepatobiliary Pancreat Sci 22: 363-370, 2015.

3 Gurusamy KS, Pamecha V, Sharma D and Davidson BR: Techniques for liver parenchymal transection in liver resection. Cochrane Database Syst Rev 1: CD006880, 2009.

4 Scatton O, Brustia R, Belli G, Pekolj J, Wakabayashi G and Gayet B: What kind of energy devices should be used for laparoscopic liver resection? Recommendations from a systematic review. J Hepatobiliary Pancreat Sci 22: 327-334, 2015.

5 Pamecha V, Gurusamy KS, Sharma D and Davidson BR: Techniques for liver parenchymal transection: a meta-analysis of randomized controlled trials. HPB (Oxford) 11: 275-281, 2009.

6 Rahbari NN, Koch M, Schmidt T, Motschall E, Bruckner T, Weidmann K, Mehrabi A, Büchler MW and Weitz J: Metaanalysis of the clamp-crushing technique for transection of the parenchyma in elective hepatic resection: back to where we started? Ann Surg Oncol 16: 630-639, 2009.

7 Aragon RJ and Solomon NL: Techniques of hepatic resection. J Gastrointest Oncol 3: 28-40, 2012.
8 Hoffmann K, Müller-Bütow V, Franz C, Hinz U, Longerich T, Büchler MW and Schemmer P: Factors predictive of survival after stapler hepatectomy of hepatocellular carcinoma: a multivariate, single-center analysis. Anticancer Res 34: 767-776, 2014.

9 Holmin T and Zilling T: Human liver resection with the aid of a stapling device. Anticancer Res 23: 1697-1700, 2003.

10 Aoki T, Murakami M, Koizumi T, Enami Y, Koike R, Fujimori A, Kusano T, Matsuda K, Yamada K, Nogaki K, Watanabe M, Otsuka K, Gareer H and Kato T: Skeletonization and isolation of the Glissonean and venous branches in liver surgery with an ultrasonic scalpel technology. Int Surg 100: 1048-1053, 2015.

11 Richter S, Kollmar O, Schuld J, Moussavian MR, Igna D and Schilling MK: Randomized clinical trial of efficacy and costs of three dissection devices in liver resection. Br J Surg 96: 593601, 2009.

12 Mbah NA, Brown RE, Bower MR, Scoggins CR, McMasters KM and Martin RC: Differences between bipolar compression and ultrasonic devices for parenchymal transection during laparoscopic liver resection. HPB (Oxford) 14: 126-131, 2012.

13 Bodzin AS, Leiby BE, Ramirez CG, Frank AM and Doria C: Liver resection using cavitron ultrasonic surgical aspirator (CUSA) versus harmonic scalpel: a retrospective cohort study. Int J Surg 12: 500-503, 2014.

14 Appéré F, Piardi T, Memeo R, Lardière-Deguelte $S$, Chetboun M, Sommacale D, Pessaux P and Kianmanesh R: Comparative study with propensity score matching analysis of two different methods of transection during hemi-right hepatectomy: Ultracision Harmonic scalpel versus Cavitron Ultrasonic Surgical Aspirator. Surg Innov 24: 499-508, 2017.

15 Dindo D, Demartines N and Clavien PA: Classification of surgical complications: a new proposal with evaluation in a cohort of 6336 patients and results of a survey. Ann Surg 240: 205-213, 2004.

16 Ishii M, Mizuguchi T, Harada K, Ota S, Meguro M, Ueki T, Nishidate T, Okita K and Hirata K: Comprehensive review of post-liver resection surgical complications and a new universal classification and grading system. World J Hepatol 6: 745-751, 2014.

17 Jin S, Fu Q, Wuyun G and Wuyun T: Management of posthepatectomy complications. World J Gastroenterol 19: 79837991,2013

18 Huntington JT, Royall NA and Schmidt CR: Minimizing blood loss during hepatectomy: a literature review. J Surg Oncol 109: 81-88, 2014.

19 Koch M, Garden OJ, Padbury R, Rahbari NN, Adam R, Capussotti L, Fan ST, Yokoyama Y, Crawford M, Makuuchi M, Christophi C, Banting S, Brooke-Smith M, Usatoff V, Nagino M, Maddern G, Hugh TJ, Vauthey JN, Greig P, Rees M, Nimura Y, Figueras J, DeMatteo RP, Büchler MW and Weitz J: Bile leakage after hepatobiliary and pancreatic surgery: a definition and grading of severity by the International Study Group of Liver Surgery. Surgery 149: 680-688, 2011.

Received March 20, 2018

Revised April 19, 2018

Accepted April 20, 2018 